\title{
Cooperação, Competição e Individualismo em uma Perspectiva Sócio-cultural Construtivista
}

\author{
Marilícia Witgler Antunes Palmieri ${ }^{12}$ \\ Universidade Estadual de Londrina \\ Angela Uchoa Branco ${ }^{3}$ \\ Universidade de Brasilia
}

\begin{abstract}
Resumo
Examinando questões de relevância e interesse no campo da psicologia, o artigo focaliza a necessidade de compreender o domínio da motivação social, destacando a questão da cooperação, competição e individualismo. A perspectiva teórica adotada valoriza os aspectos sócio-culturais, afetivos, cognitivos na análise das diferentes modalidades de interdependência humana. Resgata questões conceituais e teóricas referentes aos diversos elementos envolvidos no sistema motivacional do indivíduo, assumindo uma visão sistêmica e holística, que enfatiza a dimensão interativa e contextual presente nos processos de desenvolvimento de crenças e valores, bem como na promoção de padrões de interação social específicos. Este artigo atribui especial ênfase ao estudo e à aplicação dos conceitos básicos decorrentes da adoção de uma perspectiva sócio-cultural construtivista do desenvolvimento humano, destacando as dimensões metodológicas e ressaltando a necessidade urgente da psicologia de investigar a questão, tendo em vista a relevância do tema para a sociedade contemporânea.

Palavras-chave: Cooperação; competição; individualismo; valores; motivação social.
\end{abstract}

\section{Cooperation, Competition and Individualism from a Sociocultural Constructivist Perspective}

\begin{abstract}
In the present article we argue for the need to develop a conceptual and theorical approach to the study of social motivation, particularly focusing upon the issue of cooperation, competition and individualism. The perspective herein adopted takes into account sociocultural, affective and cognitive dimensions of human social interdependence. We discuss the multiple and diverse components of human motivational system from a systemic and holist viewpoint that emphasizes the contextual and interactive dimensions of developmental processes of value co-constructions, as well as specific patterns of social interactions. The article highlights the need to investigate the topic from a sociocultural constructivist perspective, addressing the methodological issues and, especially, stressing the urge of scientific psychology to contribute to contemporary society by providing a better understanding of processes implied in the development of cooperative, competitive and individualist values.

Keywords: Cooperation; competition; individualism; values; social motivation.
\end{abstract}

No contexto dos estudos sobre motivação social e valores humanos, muito se discute sobre categorias relativas a diferentes modalidades de participação ou interdependência social, tais como a cooperação, competição, individualismo, coletivismo, altruísmo e agressão, entre outros. Seguindo modelos teóricos específicos, muitos autores tem apresentado variadas propostas teóricas para a explicação dos motivos pelos quais as pessoas orientam suas relações umas com as outras de diferentes maneiras, enfatizando diversos aspectos e focalizando níveis diferenciados de análise.

O principal objetivo deste artigo é examinar a questão da motivação social, particularmente no que se refere às categorias de cooperação, competição e individualismo, no contexto de uma orientação teórica mais ampla, aqui

\footnotetext{
${ }^{1}$ A autora agradece a CAPES, pela bolsa de doutorado junto ao Programa de Pós Graduação em Psicologia da UnB - Brasília.

${ }^{2}$ Endereço para correspondência: Rua Joaquim Távora, 392, Jardim Sabará, Londrina, Paraná, 86066 020. Fone: (43) 33380464, Fax (43) 33381108. E-mail: marilicia@uel.br

${ }^{3}$ A autora agradece ao CNPQ pela bolsa pesquisador.
}

caracterizada como sócio-cultural construtivismo. Esta perspectiva inclui componentes sócio-culturais, afetivos e cognitivos que são articulados de forma interdependente para a análise dos processos envolvidos na dimensão motivacional do indivíduo. A questão central consiste em trazer contribuições à uma compreensão sistêmica e integrada do fenômeno da motivação social, explicitando os múltiplos aspectos envolvidos no desenvolvimento de crenças e valores, que por sua vez levam a categorias de interação social específicas, associadas à cooperação, competição e individualismo.

Para empreender investigação e análise da questão de forma a considerar os processos situados em nível macro e micro, envolvidos na co-construção de valores e no desenvolvimento de padrões de interação social específicos, será aqui adotada uma abordagem histórica, sistêmica e sóciocultural construtivista para o estudo desenvolvimento humano (Ford \& Lerner, 1992; Rogoff, 1990; Shweder, 1990; Valsiner, 1989, 1998; Wertsch, 1998). Em particular, será analisado o conceito de internalização (Lawrence \& Valsiner, 1993) no sentido de recuperar as diferentes proposições 
conceituais apresentadas por diversas teorias, destacandose aí a perspectiva sócio-cultural construtivista. Além disso, será discutida a necessidade urgente das ciências se posicionarem acerca da questão (Morin \& Prigogine, 2000), a psicologia aí assumindo sua especificidade no estudo do desenvolvimento dos valores humanos.

\section{A Questão Teórica e Conceitual da Motivação Social: Cooperação, Competição e, Individualismo}

$\mathrm{O}$ desenvolvimento de estudos que exploram o repertório complexo das relações existentes entre fenômeno psicológico e contexto resgatam a inter-relação dos aspectos culturais, afetivos, cognitivos e sociais envolvidos na construção da subjetividade humana. Tais estudos tem levado à produção de conhecimento acerca de fenômenos relativos ao desenvolvimento social (Ex.: Branco, 1998, 2001; Corsaro, Gaskins \& Miller, 1992; Eckerman \& Peterman, 2001; Eisenberg \& Mussen, 1989; Schweder, 1990; Staub, 1989, 1991; Tappan, 1992; Valsiner, 1987;1989, 1998).

Os estudos que abordam as diferentes modalidades de interdependência humana nas ciências sociais e, de forma particular na psicologia, por sua vez, têm utilizado diferentes definições conceituais e sugerido explicações diversas para o fenômeno da motivação social, apoiados em orientações teóricas e níveis de análise diversificados.

$\mathrm{Na}$ psicologia, em especial na psicologia do desenvolvimento, a discussão teórica e conceitual da cooperação e competição vem sendo considerada no nível das ações ou comportamentos observáveis, isto é, estudos têm sido realizados para investigar a ocorrência de comportamentos caracterizados como pró ou anti-sociais (Ex.: Brownell \& Carriger,1990; Eisenberg \& Mussen, 1989; Hoffman, 1990; Staub, 1989, 1991). Para a maioria dos autores, comportamentos pró-sociais são aqueles que representam ações ou atividades consideradas como socialmente positivas, visando atender às necessidades e ao bem-estar de outras pessoas, como, por exemplo, o altruísmo, a generosidade, a cooperação, os sentimentos de empatia e simpatia, etc. Por outro lado, comportamentos anti-sociais incluem ações ou atividades consideradas como socialmente negativas, voltadas, por exemplo, à destruição ou ao prejuízo de outras pessoas, e relacionadas a comportamentos egoístas, competitivos, hostis e agressivos.

Aprofundando tópicos importantes relativos à cooperação e à competição, os estudos no âmbito da psicologia do desenvolvimento também associam o nível de análise comportamental a uma dimensão motivacional e de caráter valorativo (Edwards, 1991; Eisenberg \& Mussen, 1989; RadkeYarrow \& Zahn-Waxler, 1991; Staub, 1989, 1991). Eisenberg e Mussen (1989), por exemplo, ressaltam diferenças conceituais ao tratar as subcategorias do comportamento pró-social, categoria mais ampla que inclui, segundo os autores, tanto ações altruístas, como ações motivadas por interesses específicos por parte daquele que beneficia o outro social. Destacam, portanto, o altruísmo como um construto que se caracteriza pela presença de um elemento motivacional interno, isto é, o indivíduo é motivado a atuar de forma voluntária visando o benefício de outras pessoas, sem a perspectiva aparente de ganhos pessoais, à exceção da auto-recompensa.

Para Edwards (1991), a cooperação e a competição constituem aspectos de um mesmo fenômeno relacional, a depender do contexto e do valor adaptativo de cada tipo de ação. Para o autor, cujas idéias são compartilhadas por outros autores de orientação evolucionária (Ex.: Krebs, 1996; La Frenière \& MacDonalds, 1996), ambos os comportamentos estão a serviço de objetivos individuais que vão sendo constituídos em contextos grupais determinados, que ora favorecem a cooperação, ora a competição. Desta forma, o indivíduo estará sempre maximizando suas possibilidades de adaptação ao ambiente, caracterizado por uma cultura ou situação específica. É assim que a vivência de situações cooperativas pode favorecer a expressão de comportamentos de natureza pró-social, conduzindo o indivíduo a se relacionar positivamente em relação às necessidades e bem-estar de outras pessoas, da mesma forma que contextos competitivos convidam os indivíduos à hostilidade e à agressão (Mead, 1937; Radke-Yarrow \& Zahn-Waxler, 1991).

Staub $(1989,1991)$, cuja proposta apresenta vários componentes partilhados com uma perspectiva sócio-cultural construtivista, sugere que os valores sociais encontram-se dinamicamente organizados e hierarquicamente integrados no sistema motivacional da pessoa, aí incluindo outros elementos tais como, por exemplo, orientações para objetivos pessoais (personal goal orientations), necessidades, preferências e motivos conscientes e inconscientes, bem como formas internalizadas de normas, regras e hábitos específicos da cultura. Os valores sociais representam, para o autor, importante parte do sistema complexo da motivação social, pois os valores tendem a orientar e promover comportamentos e interações específicos ao longo do processo de socialização.

No campo da psicologia social, é preciso destacar a importante contribuição do estudo clássico de Deutsch (1949). Ele dá especial ênfase ao nível estrutural e contextual na promoção dos diversos processos de interação ou formas de participação no grupo. Além disso, Deutsch propõe a existência de dois níveis articulados de análise: o da estrutura e o da subjetividade. Define a cooperação como o contexto interativo em que as ações de um participante favorecem o alcance do objetivo de ambos, sendo a competição caracterizada como a busca de objetivos mutuamente 
exclusivos, ou seja, quanto mais um indivíduo se aproxima de seu objetivo, mais o outro se afasta da possibilidade de alcançar o seu. Para ele, situações cooperativas ou competitivas se encontram em consonância com objetivos expressos nas interações em diferentes perspectivas e dimensões, ou seja, a estrutura favorece motivações individuais subjetivas a ela semelhantes (nível da subjetividade). Isto significa que contextos cooperativos tendem a facilitar ou promover dinâmicas interacionais cooperativas, mas não necessariamente, porque as pessoas podem interagir em desacordo com as regras do jogo se, em termos de motivação pessoal, estiverem orientadas em outra direção. Além disso, Deutsch destaca que normas e regras de natureza cooperativa e competitiva estabelecidas por um grupo social podem ser estruturadas dentro de um único contexto complexo, o qual organiza diferentes situações de relacionamento entre os indivíduos. Este seria o caso, por exemplo, da cooperação intra-grupo associada à competição inter-grupo.

A análise de Deutsch(1949) contribui, particularmente, para chamar a atenção para a importância do contexto, com suas regras e expectativas sociais. $\mathrm{Na}$ versão sócio-cultural construtivista, tal processo é designado como canalização cultural (Valsiner, 1998), como veremos adiante. O autor, entretanto, não dá a devida importância ou destaque, em sua análise, ao caráter processual do desenvolvimento humano em sua dimensão semiótica e sistêmica, o que é necessário para abranger a complexidade do fenômeno da interdependência social em seus aspectos macro (históricocultural), micro (contextual/situacional) e subjetivo (individual), os quais estão em permanente processo de mudança ou transformação ao longo da dimensão temporal (Branco,1998).

O fenômeno da motivação precisa ser analisado e entendido, portanto, em sua profunda conexão com o contexto sócio-cultural, superando as tradições da psicologia social (que, mais modernamente, valoriza o contexto) e da psicologia do desenvolvimento clássica (Freud e Piaget, que valorizam particularmente o sujeito e seus motivos pessoais). É fundamental destacar a idéia de interdependência pessoacontexto, dando ênfase às articulações e relações dialógicas e complexas entre as partes envolvidas. Acreditamos ser de extrema importância analisar de forma integrada, ou, ainda melhor, de forma sistêmica, os diversos aspectos do desenvolvimento de valores e da dinâmica das interações, em virtude do conjunto de experiências vivenciadas pelo sujeito nos múltiplos espaços sociais dos quais participa. São tais experiências e práticas que configuram variados processos de atribuição de significado às ações humanas. A existência de opções, preferências, motivos e convicções nos indivíduos, expressos através de comportamentos mediados semioticamente na relação do eu com o outro, exigem que a psicologia adote um posicionamento teórico e metodológico que lhe permita investigar mais especificamente a dinâmica dos processos desenvolvimentais responsáveis pela co-construção dos sistemas motivacionais dos sujeitos em interação.

Entendemos que considerar a simples manifestação comportamental ou a emissão de opiniões ou preferências pessoais através da aplicação de questionários, de forma isolada, implica em uma séria dificuldade de compreender a questão em sua complexidade. É necessário, pois, aprofundar o estudo no sentido de articular os diferentes aspectos manifestos em nível comportamental, relacional e estrutural-contextual, dentro do contexto mais amplo da sócio-cultura (Branco, 2001).

Partimos da premissa de que o universo motivacional do sujeito envolve crenças, valores e orientações para objetivo que sustentam suas ações nas interações com outras pessoas. Dependendo da disposição motivacional do sujeito e do contexto em que se insere, a probabilidade de ações cooperativas, competitivas e/ou individualistas se altera de forma significativa. E é nesse sentido que aqui destacamos a importância da motivação social como fenômeno relevante a ser estudado, devido à sua centralidade com relação ao desenvolvimento da personalidade, dos processos de identidade e das conseqüências sociais em termos da coconstrução entre sujeito e cultura, indivíduo e sociedade, polaridade esta que precisa ser encarada como um sistema holístico a definir-se de forma dinâmica e contínua ao longo do tempo (Magnusson, 1995). A motivação social, assim concebida, pode ser definida, portanto, como o conjunto dinâmico e hierarquizado de crenças, valores, metas e objetivos associados a diferentes padrões de interação social relacionados a prática da cooperação, competição e individualismo em um contexto sócio-cultural determinado.

O primeiro passo na investigação da motivação social consiste, então, em reconhecer e analisar sob diferentes ângulos, a heterogeneidade da motivação humana e dos contextos culturais nos quais os indivíduos se circunscrevem (Branco, 1996; Branco \& Valsiner, 1997; Eisenberg \& Mussen, 1989; Mead, 1937; Triandis, 1991).

De fato, grande é a variabilidade cultural relativa a valores e comportamentos sociais no estudo da cooperação, competição e individualismo (Eisenberg \& Mussen, 1989). Em uma dimensão antropológica, o estudo de Mead (1937) constitui-se em um exemplo clássico das diferentes modalidades de interdependência humana expressas no âmbito da cultura, muito embora existam dificuldades metodológicas em seu trabalho, decorrentes da adoção de concepções e categorias de análise pré-determinadas: a autora já inicia o seu estudo com base nas categorias 
previamente definidas de cooperação, competição e individualismo. Examinando diferentes modalidades de participação social, Mead (1937) revela e discute o caráter cultural substancialmente diverso e heterogêneo com que os indivíduos de 13 diferentes sistemas sociais se organizavam, expressando padrões interacionais diferenciados de natureza cooperativa, competitiva e individualista. Apesar da rica complexidade de seus dados e análises, entretanto, esta tende a ficar obscurecida diante da classificação das culturas e da utilização de categorias psicanalíticas pré-estabelecidas, que dificultam a análise das culturas em sua originalidade e complexa heterogeneidade, objetivo principal da antropologia contemporânea.

Em se tratando da análise de padrões culturais marcados pelo individualismo e pelo coletivismo, encontramos nos estudos de Triandis $(1991,1995)$ a cooperação entendida como um dos atributos característicos de grupos sociais coletivistas, onde a motivação individual sistematicamente se refere ao grupo de pertencimento constituído pela família, tribo, ou nação. Já nas culturas por ele classificadas como individualistas, as pessoas tenderiam mais à competição e à defesa de seus interesses particulares. O mais importante, porém, é analisar a questão da motivação social em sua complexidade, evitando dicotomias que não consideram a riqueza da subjetividade humana em dinâmica relação com o contexto histórico sócio-cultural complexo, ambos em permanente desenvolvimento (Branco, 1996).

Em resumo, o caráter dinâmico e dialógico que caracteriza o domínio da motivação social precisa ser sempre considerado, expressando, assim, a necessidade de não se reduzir as explicações relativas às diversas formas de participação social a princípios exclusivamente individualistas ou coletivistas. A noção de sistemas classificatórios e puramente descritivos, muitas vezes encontrada na literatura, é incompatível com o estudo da motivação social no contexto de uma orientação sócio-cultural construtivista, uma vez que esta não adota categorias universais e estáveis na explicação dos fenômenos desenvolvimentais.

A princípio, lidar com dualidades ou dicotomias pode representar um caminho viável para a reflexão dos aspectos envolvidos no estudo da motivação social, auxiliando-nos até mesmo a melhor compreender a natureza da relação que se configura em diferentes momentos e situações ligadas a vida motivacional do sujeito. O problema se coloca, porém, quando tendemos a reconhecer orientações positivas ou prósociais (como o altruísmo, cooperação, simpatia, generosidade, etc.) de forma universalista e não contextualizada. $\mathrm{O}$ mesmo ocorre quando consideramos orientações de natureza individualista como sempre negativas, pois conduziriam o indivíduo a atuar em benefício próprio em detrimento dos demais, ou seja, como se tais ações necessariamente conduzissem à competição, egoísmo, hostilidade, agressão, etc. Tal visão maniqueísta claramente desconsidera os significados culturais e a complexidade do fenômeno humano, deixando aberta a possibilidade para generalizações etnocêntricas ou culturalmente enviesadas.

O tema da motivação social apresenta, em suas múltiplas manifestações, a possibilidade de dialogarmos com diversas tendências teóricas marcadas semioticamente pelo momento histórico-cultural e pelas configurações típicas dos grupos humanos em que é investigado. O modo de produção capitalista do mundo ocidental, por exemplo, parece configurar, em si mesmo, uma base de sustentação para diferentes versões de individualismo. Pode-se afirmar, em acordo com Dumont (1985), que o individualismo enquanto categoria vem se configurando ao longo de transformações históricas causadas pelo advento do Cristianismo e, mais recentemente, da Revolução Industrial do século XVIII. À medida em que a humanidade passou a caminhar em direção ao avanço tecnológico, associado ao liberalismo econômico típico da evolução e consolidação do capitalismo, novas relações, necessidades e interesses foram sendo constituídos entre os seres humanos (Jurberg, 2000; Triandis, 1991; Velho, 1987). O modelo de produção capitalista centraliza-se na disposição competitiva e no controle organizacional dos modos de pensar, perceber, sentir, relacionar-se e, em particular, na existência de equipamentos coletivos que se articulam no processo de produção (Silva, 1999). Além disso, o capitalismo também suscita um novo tipo de agrupamento social: o coorporativismo, onde pessoas pertencentes a uma mesma categoria são motivadas a agir em torno de interesses e objetivos comuns.

A tendência das pessoas a se identificarem com grupos sociais específicos no sistema de produção capitalista, tal como descrito por Jurberg (2000), revela como uma atuação individualista pode promover uma forma de vida social pautada em relações coorporativistas. Esta posição tende a levar os indivíduos, mesmo de maneira não proposital, a se relacionarem uns com os outros de uma forma pseudocooperativa, fazendo-os representar ideologias que os aprisionam a seus objetivos, orientado-os para direções específicas (Guareschi, 1999). Como resultado, formas coorporativas de expressão podem orientar pessoas e instituições no sentido da legitimação de preconceitos, julgamentos prévios entre os pares e tratamentos diferenciados, restringindo o desenvolvimento de motivações mais pró-sociais e interações mais amplamente cooperativas, caracterizadas por sentimentos de empatia e respeito mútuo entre os seres humanos (Jurberg, 2000).

Em um estudo abrangente e complexo, Gilberto Velho (1987) mostra a sociedade moderna e atual como favorecedora de possibilidades de individualização. Para ele, 
enquanto um processo gerado dentro de regras e padrões sociais, a hierarquia de valores e o contexto sócio-cultural funcionam como agentes facilitadores, em maior ou menor grau, da produção e manutenção do processo de individualização. Para Velho (1987), tal como para Dumont (1985), as relações humanas, hoje, trazem consigo uma marca essencial que cristaliza a própria ideologia moderna, sob o fermento do individualismo. Nesta direção, ações individualistas podem ser pensadas em termos da promoção de orientações sociais e disposições psicológicas, que se caracterizam pela disposição da pessoa em orientar-se exclusivamente para o próprio bem-estar, em detrimento dos demais e da coletividade, desconsiderando a repercussão que tal disposição representa na relação com outras pessoas ou grupos sociais. É necessário, porém, distinguir processos de individuação e individualismo, pois o primeiro representa a conquista e o reconhecimento da pessoa em sua condição de originalidade, autonomia e liberdade, e o segundo relaciona-se de perto com disposições egoístas, coorporativistas, hostis e competitivas.

A sociedade se constitui em rica fonte de esteriótipos e manipulações, configurando sistemas valorativos que influenciam os tipos de comportamentos avaliados pelo indivíduo como positivos ou negativos (Martinéz, 1997). Entretanto, na integração dos diferentes elementos envolvidos na interdependência humana não podemos desconsiderar a produção de diferentes significados para determinadas ações, os quais devem ser sempre entendidos no âmbito de contextos culturais específicos, levando-se em conta a dimensão da subjetividade dos indivíduos em interação. Cada pessoa vivencia diferentes situações, produzindo diferentes significados e gerando novidades psicológicas, que por sua vez podem configurar mudanças nos contextos sócio-culturais em que atuam.

A abordagem sócio-cultural construtivista, portanto, se constitui em uma opção teórica promissora, ao apresentar uma concepção de desenvolvimento humano que leva em conta as dimensões semióticas e subjetivas expressas tanto em nível do contexto, quanto do indivíduo, este último entendido como agente de seu próprio desenvolvimento (Ex: Branco \& Valsiner, 1997; Bruner, 1996; Shweder, 1990; Valsiner, 1989, 1998).

\section{Desenvolvimento Humano na Perspectiva Sócio- Cultural Construtivista}

O desenvolvimento humano pode ser concebido a partir de uma perspectiva unilinear ou de uma perspectiva de complexidade (Morin, 1992; Valsiner, 1989, 1998). A unilinearidade de uma perspectiva desenvolvimental parte do pressuposto de que as pessoas seguem uma trajetória única e universal no percurso de seu desenvolvimento.
Neste caso, prevalece a noção de uma sequenciação de estágios desenvolvimentais pré-determinados, os quais o sujeito supostamente percorre em uma sequência processual (visão teleológica de desenvolvimento). Valsiner (1989) se refere a esta visão como sendo a adotada pela maioria dos cientistas na área, que visualizam o desenvolvimento como atrelado a esquemas de programação genética, mesmo quando a idéia de interação indivíduo-ambiente é levada em conta. Segundo os modelos tradicionalmente unilineares, pouca ênfase tem sido dada ao caráter dinâmico e complexo dos processos desenvolvimentais, bem como ao papel constituinte da cultura, em que esta não é vista como variável externa a ser estudada, mas como componente coestruturador do desenvolvimento humano (Cole, 1992).

A perspectiva multilinear (também denominada como sistêmica ou complexa) reconhece a possibilidade de múltiplas trajetórias no processo de desenvolvimento. Desse ponto de vista, as transformações que ocorrem ao longo do desenvolvimento do sujeito não se dão de forma unilateral - ora no organismo, ora no ambiente - mas na relação de interdependência recíproca que se estabelece entre eles.

Ao sublinhar o papel da cultura na constituição dos processos desenvolvimentais, entretanto, é fundamental enfatizar os perigos inerentes à afirmação de que todas as funções ou características psicológicas dos seres humanos são socialmente construídas ou determinadas. Tal afirmação traz, com frequência, marcas que descartam a qualidade dialética e processual da participação ativa do sujeito em desenvolvimento no contexto sócio-cultural no qual este se insere. Se tomada de forma isolada, tal afirmação, igualmente reducionista, impossibilita encontrar respostas aos verdadeiros problemas decorrentes de uma visão sóciogenética dos fenômenos psicológicos (Valsiner, 1994).

Segundo a abordagem aqui adotada, é necessário que o caráter dialógico da interdependência indivíduo-sociedade seja postulado, sendo preciso, então, investigar os mecanismos específicos pelos quais o contexto sócio-cultural exerce sua influência (moral, política, religiosa, artística, etc.) sobre o sujeito, e vice-versa. Ou seja, é imprescindível investigar de que forma ambos, contexto e indivíduo, constituem-se como participantes ativos na constituição da subjetividade do ser em desenvolvimento.

O termo separação inclusiva, tal como utilizado por Valsiner e Cairns (1992) em sua discussão acerca da noção de conflito, permite uma compreensão mais adequada da natureza da relação entre Indivíduo e Sociedade. A idéia de separação inclusiva contempla a diferenciação entre a pessoa e o seu mundo social, mas implica na superação dialética de dualismos, ao afirmar a dualidade sem perder a noção do todo constituído pelo sistema de relações que se estabelecem entre as partes. A utilização desse construto é 
particularmente útil para analisarmos a questão das diferentes modalidades de interdependência social, buscando identificar os componentes e processos sistêmicos envolvidos na dinâmica das interações indivíduo - sociedade.

Assumir a separação inclusiva e a concepção processual de desenvolvimento enquanto construtos analíticos conduz, assim, a questionar toda e qualquer tipologia baseada em traços e características permanentes, ou categorias mutuamente exclusivas no campo da psicologia. Isto permite a elaboração de novas questões teóricas e metodológicas que irão representar os atuais desafios da psicologia contemporânea.

Também julgamos fundamental aqui ressaltar a importância da dimensão tempo na concepção de desenvolvimento segundo a visão sócio-cultural construtivista. A dimensão temporal implica na idéia de contínuas transformações qualitativas, dinâmico-estruturais, que se dão ao longo da trajetória de desenvolvimento da pessoa. O desenvolvimento, então, ocorre no tempo, e mediante um processo de transformação que resulta de complexas inter-relações que se estabelecem entre o sujeito e o ambiente sócio-cultural, processo este que também é orientado para o futuro (Valsiner, Branco \& Dantas, 1997). O importante dessa concepção integrada de passadopresente-futuro representa, principalmente, a abertura de um espaço para a indeterminação dos processos desenvolvimentais, onde a construção e a emergência do novo se torna possível.

Quando focalizamos o mundo subjetivo da pessoa, vemos este sendo construído e reconstruído constantemente a partir das relações dinâmicas e de contínua transformação que ela estabelece com o mundo externo e objetivo, culturalmente mediado. Destacam-se, assim, o caráter ativo do sujeito na construção de seu próprio desenvolvimento, bem como a participação efetiva das sugestões sociais presentes nos mecanismos de canalização cultural que orientam os limites físicos e semióticos que atuam nos processos interativos entre indivíduo e contexto (Valsiner, 1998; Valsiner \& cols., 1997). Os conceitos de canalização cultural e de participação ativa do sujeito representam a base teórica mais importante do sócio-cultural construtivismo.

No caso das interações sociais, o poder da canalização cultural é evidenciado por inúmeros estudos e evidências científicas nas áreas das ciências humanas e sociais, particularmente da psicologia (ver Branco, 1998; Triandis, 1991). Ambientes estruturados de forma cooperativa tendem a gerar cooperação, enquanto ambientes estruturados de forma individualista ou competitiva favorecem claramente a emergência de padrões de hostilidade e competição (ver revisão de Johnson \& Johnson, 1989). A existência de tais processos, portanto, representa o aspecto de determinação relativa (dialeticamente relacionada ao indeterminismo) dos fenômenos desenvolvimentais.

Outro construto central, de grande relevância para o tema aqui analisado, é o de internalização, pois este se encontra na própria origem dos processos desenvolvimentais envolvidos na co-construção de valores.

\section{Explorando o Conceito de Internalização}

O conceito de internalização tem sido utilizado por diversos autores na tentativa de explicar o processo dinâmico através do qual se torna possível compreender como conteúdos e processos inter-psicológico são trazidos ao plano intra-psicológico. $\mathrm{Na}$ teoria psicanalítica, o conceito de identificação se refere à idéia de que o sujeito incorpora a base sócio-moral da estrutura social (valores, idéias, conceitos e outras formas de material psicológico) a partir dos vínculos afetivos que emergem das relações estabelecidas com os outros, especialmente os pais (e ainda mais especificamente, $o$ pai). No pensamento sócio-genético de Pierre Janet (Valsiner, 1998; Van der Ver \& Valsiner, 1988) o conceito de internalização também ocupa um lugar central. Volta-se, basicamente, à compreensão do funcionamento dinâmico das funções psicológicas do indivíduo, embora estas apresentem diferentes graduações em termos de valor. Janet postula que, internamente, o sujeito organiza de forma dinâmica e hierárquica suas funções psicológicas, ao mesmo tempo em que as reorganiza ao longo de seu desenvolvimento e das interações que estabelece com a realidade externa e objetiva.

Em James Baldwin, psicólogo americano do início do século XX, contemporâneo de Janet, encontramos contribuições relativas a uma compreensão dos processos de transformação inerentes ao funcionamento psicológico do indivíduo, quando ele enfatiza o papel ativo do sujeito na construção de seu mundo interno e subjetivo (Valsiner, 1994). Para explicar como os mecanismos de seleção externa (plano social) tornam-se internos (plano pessoal), Baldwin introduz o conceito de imitação persistente, que é apresentado como um mecanismo transformacional através do qual a singularidade do sujeito vai sendo construída sob o fluxo das contínuas reconstruções feitas pelo indivíduo com base nos modelos disponibilizados pelo contexto da sócio-cultura ao longo do curso de seu desenvolvimento. O conceito de internalização é também encontrado em George Mead (Valsiner, 1998; Van der Ver \& Valsiner, 1988), para quem a construção do self se dá mediante um processo construtivo nos domínios intrapessoal e interpessoal. Através do circuito denominado $I \leftrightarrow \rightarrow M e$ [grifo nosso] como representação da instância agente $\leftarrow \rightarrow$ instância reflexiva, Mead se apóia na existência de uma reconstrução interna e ativa do mundo pessoal - identificada com o sujeito I , a qual vai gerando 
experiências subjetivas novas, assim como novas ações, que acabam por transformar, ao mesmo tempo, o ambiente e o sujeito. Assim, o I (sujeito agente) torna-se Me (sujeito reflexivo), na medida em que reorganiza subjetivamente o material psicológico proveniente de experiências socialmente compartilhadas. O Me, por sua vez, a cada momento, constitui-se objeto de análise do I.

É, porém, nas formulações de Vygotsky sobre a idéia de internalização que encontramos uma elaboração mais sofisticada do conceito. Vygotsky, particularmente interessado na natureza co-construtiva do desenvolvimento cognitivo (1930-1994; 1986), reconhece a internalização como a interiorização de conteúdos históricos determinados e culturalmente organizados. Ao lidar com as relações afetas à linguagem e à complexidade das funções psíquicas superiores (particularmente o pensamento), Vygotsky associa a idéia de que o processo de interiorização envolve a reconstrução da atividade psicológica interna, tendo como base as operações com signos externos, especialmente os signos lingüísticos. Ao postular que os signos lingüísticos operam como mediadores da re-elaboração mental de processos que transformam o inter-subjetivo em intrasubjetivo, Vygotsky reconhece o papel intrumental da linguagem na reconstrução mental dos recursos internos de que o sujeito dispõe frente a eventos que ocorrem na realidade externa (na resolução de problemas, na tomada de decisões, etc.). Enfim, este movimento de fora (interpsicológico) para dentro (intra-psicológico) é entendido na teoria histórico-cultural como o resultado das experiências compartilhadas pelo sujeito com outras pessoas, gerando uma série de transformações de natureza qualitativa nas funções psicológicas no percurso do desenvolvimento individual. Suas idéias sobre o conceito de internalização é que servirão de base para a elaboração do mesmo na perspectiva ora adotada.

\section{Internalização e Externalização na Perspectiva Sócio- Cultural Construtivista}

O conceito de internalização se afigura como central no contexto de uma orientação sócio-cultural construtivista. Internalização e externalização consistem, em uma perspectiva de separação inclusiva, processos de coconstrução que se dão no espaço intersubjetivo das interações sociais configurando, em última análise, a contínua troca / criação / comunicação de mensagens culturais entre os indivíduos em interação. Consistem na co-construção de significados produzidos por processos comunicativos que ocorrem tanto no nível interpessoal, como no nível intrapessoal. A internalização direciona-se do inter para o intra, enquanto a externalização caminha na direção inversa, sendo ambos os processos, porém, intrinsicamente relacionados. Envolvem mecanismos que estruturam e dinamizam a troca e mútua elaboração construtiva que se dá entre a cultura pessoal - relativa à subjetividade - e a cultura coletiva - âmbito dos significados partilhados pelo grupo (Lawrence \& Valsiner, 1993; Valsiner, 1987, 1989, 1998).

Valsiner $(1994,1998)$ utiliza os termos cultura pessoal e cultura coletiva para expressar as instâncias do sujeito e do social, em interação constante, constituindo o fenômeno psicológico. A cultura coletiva representa os significados compartilhados pelos grupos de referência dos quais o sujeito participa, incluindo-se aí a linguagem, as normas sociais e as práticas de vida cotidiana. A cultura pessoal se refere a um sistema constituído por um processo permanente de internalização reconstrutiva de normas, valores, crenças e opiniões, enfim, de significados transformados através do contato com a cultura coletiva que é, por sua vez, ativamente processada pelo indivíduo de forma única e singular. Nos contextos comunicativos a cultura pessoal é externalizada, dando origem a diferentes significados ao introduzir novos elementos à cultura coletiva. $\mathrm{O}$ essencial aqui é entender que as dimensões da subjetividade e do espaço social tem um papel constituinte no processo de co-construção do desenvolvimento individual e cultural.

Do ponto de vista do indivíduo, a internalização dos conteúdos culturais é orientada por múltiplos fatores motivacionais, afetivos etc, que selecionam e priorizam objetivos, metas e aspectos culturais (não necessariamente de forma intencional), atribuindo-lhes um significado próprio a partir de um amplo universo de possibilidades. A dinâmica social de produção de significações envolve, portanto, uma multiplicidade de sentidos pessoais. É importante ressaltar aqui que, devido ao caráter dinâmico da produção de significados, evitamos distinguir significado de sentido como o faz Vygotsky, e utilizamos a noção de significado de forma mais ampla (Bruner, 1996). Sendo assim, os significados que vão sendo produzidos nos processos interativos conduzem o indivíduo à problematização de concepções, idéias e conceitos já existentes, configurando novas formas de compreensão, motivação e atuação concreta, em termos de metas, opções, ações, relações, etc. Em função de suas características e motivações específicas, gera-se a possibilidade do indivíduo recriar de forma singular e única sistemas de significação típicos de sua subjetividade (cultura pessoal), o que poderá permitir, ao longo do tempo, o rompimento com padrões socialmente definidos de crenças, valores e comportamentos. Mediante tais processos, o sujeito poderá, inclusive, introduzir novos elementos à cultura coletiva de maneira mais ou menos extensa, gerando por vezes transformações no contexto sócio-cultural que transcendem a dimensão da família ou grupo de convivência. 
Analisando contextos de interação entre adultos e crianças, podemos dizer que as mensagens culturais ativamente comunicadas pelos adultos são ativamente processadas pela criança, através de processos simultâneos de internalização e externalização. Crianças e adultos são construtores conjuntos de novos significados culturais e, em seus respectivos papéis, são construtores ativos e conjuntos da cultura coletiva (Valsiner \& cols., 1997). Assim, podemos afirmar, juntamente com Lawrence e Valsiner (1993), que os valores culturais e padrões sociais são permanentemente submetidos a processos transformacionais, na medida em que são internalizados e externalizados. Esta é a dinâmica dos processos que constituem, e continuamente modificam, valores, crenças, preferências coletivas e individuais, bem como outros produtos culturais.

Compreender a rede de significados produzidos nos processos de internalização e externalização, sob a visão teórica adotada, nos leva necessariamente a um desafio metodológico: Como especificar o quê, exatamente, é trazido para o mundo intra-psicológico? Como entender a dinâmica das transformações resultantes do diálogo interno das diferentes vozes (Bakhtin, 1986; Wertsch, 1998) no contexto das emoções co-constituídas nos processos de comunicação intersubjetiva? Como analisar as condições ou circunstâncias em que se dão os processos co-construtivos de significação, uma vez que não se trata simplesmente de uma aquisição, ou mesmo apropriação da cultura coletiva no âmbito individual (Valsiner, 1994)? Acreditamos que a adoção de metodologias qualitativas (Corsaro \& cols., 1992) e de caráter microgenético (Siegler \& Crowley, 1991) sejam particularmente produtivas na busca de respostas para tais questões.

\section{Universo Motivacional dos Valores Sociais: Cooperação, Competição e Individualismo}

Crenças e valores constituem-se em elementos que compõem o sistema da motivação social do sujeito. Cada pessoa atribui um significado pessoal e subjetivo ao conjunto das experiências vividas, bem como a seu próprio envolvimento em diversas situações. Tradicionalmente na psicologia, os conceitos de crenças e valores se referem a atributos individuais, inicialmente considerados como estáveis e consistentes, para designar concepções relativas a critérios de preferência (Khon, 1979). Entretanto, a compreensão desses conceitos articulada à uma perspectiva sistêmica e processual de desenvolvimento nos possibilita melhor expressar a dimensão co-construtiva que caracteriza o contexto das dinâmicas interativas.

Focalizando o estudo da motivação social a partir de um sistema complexo e dinâmico, a perspectiva sócio-cultural construtivista procura integrar os aspectos afetivos, cognitivos, contextuais e culturais aí envolvidos. Sendo assim, nos distanciamos de uma definição estática e universal representada pelos conceitos tradicionais de crença e valor e, nos aproximamos de uma análise conceitual que contempla as características dinâmicas do funcionamento psicológico, passando a empregar o termo orientação.

A introdução do termo orientação no contexto sóciocultural construtivista busca assegurar o caráter aberto e dinâmico dos conceitos de objetivos, crenças e valores, associados a idéia de transformação (Branco \& Valsiner, 1997). Objetivos, crenças e valores, nesse contexto, não existem de forma estática e independente, pois ao mediatizar a relação bidirecional pessoa-contexto cultural, vão se constituindo e se incorporando ao sistema motivacional da pessoa de forma contínua e transformadora, em função de perspectivas subjetivas que englobam as dimensões de passado, presente e futuro. A diferença conceitual entre crença e valor, conforme colocada por Valsiner e colaboradores (1997), dá-se principalmente em função da carga afetiva especialmente densa que está presente no conceito de valor, o qual também se articula mais proximamente com o conceito de meta ou objetivo. Motivos e convicções, crenças, valores e objetivos, portanto, se organizam de forma sistêmica, sendo tal organização sujeita a sucessivas re-estruturações em função da atuação de fatores internos e externos ao sujeito contextualizado. No caso das orientações para objetivo, estas podem ser definidas como processos dinâmicos de desenvolvimento psicológico, que envolvem um sistema de limites internos e subjetivos, semioticamente mediado, o qual, por estar direcionado ao futuro, impulsiona e limita as ações, sentimentos e pensamentos dos indivíduos no tempo presente (Branco \& Valsiner, 1997; Valsiner \& cols., 1997).

Estaremos neste artigo nos referindo particularmente ao conceito de valor, na medida em que este se afigura como um caso especial de orientação para crença que se caracteriza por maior estabilidade, devido à carga emocional e ao papel fundamental que desempenha nos processos de formação da identidade. Além disso, os valores apresentam conexão mais próxima com as orientações para objetivo, sendo assim construtos psicológicos particularmente relevantes para a compreensão do sistema da motivação social e do desenvolvimento de padrões de interação social específicos (Branco, 1998; Valsiner \& cols., 1997).

Cada pessoa vai, pois, organizando seu universo motivacional de forma subjetiva e original através de processos intencionais (mais associados à cognição e à linguagem) e não intencionais (mais associados à dimensão do afeto e das emoções). Isto ocorre à medida em que a pessoa vivencia novas experiências em contextos sucessivos. O sistema motivacional do indivíduo está constantemente 
sendo construído e reconstruído, em função de suas orientações para objetivos, crenças e valores, sendo a pessoa freqüentemente motivada por um amplo conjunto de metas e valores que vão definindo prioridades específicas que se modificam no fluxo das relações entre a pessoa e o contexto (Branco, 2001).

Em resumo, podemos dizer que o sistema motivacional da pessoa envolve orientações para crenças, valores e objetivos individuais, que acabam por participar ativamente na promoção de padrões de interação social específicos, como a cooperação, competição e individualismo.

Do ponto de vista metodológico cabe a proposta da utilização de estratégias microgenéticas para a análise das interações sociais (Branco \& Valsiner, 1997), as quais possibilitam documentar a emergência das interações que se organizam em um fluxo complexo de atividades orientadas para objetivos, que podem se apresentar ora convergentes (quando há compatibilidade de objetivos), ora divergentes (quando há incompatibilidade de objetivos), ora envolvendo processos de negociação. Além disso, permitem a identificação de padrões de interação que se apresentam ora claros, ora ambíguos ou ambivalentes. O nível microgenético de análise assegura, assim, a visualização das orientações para objetivos individuais, conduzindo a processos interacionais convergentes (Ex.: a cooperação), divergentes (Ex.: a competição), a processos de negociação ou à ambivalência.

Branco e Valsiner (1997) destacam a importância dos processos de negociação, exemplificando como processos interativos divergentes (incompatibilidade de objetivos), podem, de fato, se transformar em padrões de interação convergente (compatibilidade de objetivos). Este é apenas mais um exemplo da complexidade que envolve o estudo da motivação social e dos padrões interacionais relativos ao fenômeno da cooperação, competição e individualismo, e que convida a psicologia a encarar de forma igualmente complexa a questão.

\section{Considerações Finais}

Buscou-se, no presente artigo, sublinhar a necessidade de se adotar uma orientação teórica que contemple os elementos sócio-culturais, afetivos e cognitivos na análise de questões inerentes ao tema da motivação social, particularmente a questão da cooperação, competição e individualismo. A perspectiva sócio-cultural construtivista introduz novos conceitos e concepções que nos parecem importantes, tendo em vista a compreensão do fenômeno da motivação social de forma sistêmica e integrada, resgatando as dimensões interativa e contextual envolvidas no desenvolvimento de valores e modalidades diferentes de interação social. Ao analisar o conceito de internalização, e sua estreita relação com os processos co-construtivos que estão na base do desenvolvimento dos valores e interações sociais, nosso objetivo foi sensibilizar profissionais e pesquisadores para a importância das dinâmicas interacionais que se dão no dia-a-dia da vida do ser em desenvolvimento, e a repercussão de tais práticas, atividades e experiências no que tange a emergência e consolidação de valores e tendências comportamentais específicas. Lembramos especialmente o papel fundamental da emoção e do afeto, no que diz respeito à canalização cultural e, em consequência, a internalização de valores sociais específicos.

O artigo propõe, portanto, um referencial de análise teórica e metodológica que tem por objetivo principal permitir a investigação dos processos de elaboração inerentes à construção de valores sociais e à promoção de padrões de interação social que sejam de fato compatíveis com os objetivos estabelecidos por uma sociedade democrática e coerente com os valores humanos de igualdade, liberdade e fraternidade.

\section{Referências}

Bakhtin, M. (1986). The problem of speech genres. Em C. Emerson \& M. Holquist (Orgs.), Speech genres and other late essays (pp. 71-86). Austin: University of Texas Press.

Branco, A . U. (1996). Constraints on the universality of psychological constructs (Review essay). Culture and Psychology, 4, 477- 483.

Branco, A. U. (1998). Cooperation, competition and related issues: A coconstructive approach. Em M. C. Lyra \& J. Valsiner (Orgs.), Child development within culturally structured environments (Vol. 4, pp. 181-205). Norwood, NJ: Ablex.

Branco, A . U. (2001). Contextual, interactional and subjective dimensions of cooperation and competition: A co-constructivist analysis. Em S. Chaiklin (Org.), The theory and practice of cultural-historical psychology (pp. 107-123). Denmark: Aarhus University Press.

Branco, A. U. \& Valsiner, J. (1997). Changing methodologies: A co-constructivist study of goal orientation in social interaction. Psychology and Developing Societies, 9, 35-64.

Brownell, C. A. \& Carriger, M. S. (1990). Changes in cooperation and self-other differentiation during the second year. Child Development,61, 1164-1174.

Bruner, J. S. (1996). The culture of education. Boston, MA: Harvard University Press.

Cole, M. (1992). Culture in development. Em M. H. Bornstein \& M. E. Lamb (Orgs.), Developmental psychology: An advanced textbook ( $3^{\mathrm{a}}$ ed., pp. 731-788). Hillsdale, NJ: Lawrence Erlbaum.

Corsaro, W. A., Gaskins, S. \& Miller, P. J. (1992). Theoretical and methodological perspectives in the interpretative study of children. Em W. A. Corsaro \& P. J. Miller (Orgs.), Interpretative approaches to children's socialization (New Directions for Child Development, 58, 5-24). San Francisco, CA: Jossey-Bass.

Deutsch, M. (1949). A theory of co-operation and competition. Human Relations, $2,129-152$

Dumont, L. (1985). O individualismo: Uma perspectiva antropológica da ideologia moderna. Rio de Janeiro: Rocco.

Edwards, J. (1991). Cooperation and competition: Two sides of the same coin? The Irish Journal of Psychology, 12, 76-82.

Eisenberg, N. \& Mussen, P. H. (1989). The roots of prosocial behavior in children. New York, NY: Cambridge University Press. 
Eckerman, C. \& Peterman, K. (2001). Peers and infant social communicative development. Em G. Bremner \& A. Fogel (Orgs.), Blackwell bandbook of infant development (pp. 19-40). Oxford, UK: Blackwell.

Ford, D. H. \& Lerner, R. M. (1992). Developmental systems theory. Thousand Oaks, CA: Sage.

Guareschi, P. A. (1999). Ideologia. Em M. G. C. Jacques, M. N. Strey, M. G. Bernardes, P. A. Guareschi, S. A. Carlos \& T. M. G. Fonseca (Orgs.), Psicologia social contemporanea: Livro-texto ( $3^{\mathrm{a}}$ ed., pp. 89-103). Petrópolis, RJ: Vozes.

Hoffman, M. L. (1990). The contribution of empathy to justice and moral judgment. Em N. Eisenberg \& J. Strayer (Orgs.), Empatby and its development (pp. 47-80). Cambridge, MA: Cambridge University Press.

Johnson, D.W. \& Johnson, R.T. (1989). Cooperation and competition: Theory and research. Minnesota, MI: Interaction.

Jurberg ,M. B. (2000). Individualismo e coletivismo na psicologia social: Uma questão paradigmática. Em R. H. F. Campos \& P. A. Guareschi (Orgs.), Paradigmas em psicologia social: A perspectiva latino-americana (pp. 118-166). Petrópolis, RJ: Vozes.

Kohn, M. L. (1979).The effects of social class on parental values and practices. Em D. Reiss \& H. Hoffman (Orgs.), The american family: Dying or developing? (pp. 45-68). New York, NY: Plenum.

Krebs, D. L. (1996). The value of evolutionary perspectives on social relations among children: A commentary. International Journal of Behavioral Development, 19, 75-80.

La Frenière, P. J. \& Mac Donald, K. B. (1996). Evolutionary perspectives on children's resource-directed behaviour in peer relationships: An introduction. International Journal of Behavioral Development, 19, 1-5.

Lawrence, J.A.\& Valsiner, J.(1993). Conceptual roots of internalization: From transmission to transformation. Human Development, 36, 150-167.

Magnusson, D. (1995). Individual development: A holistic, integrated model. Em P. Moen, G. H. Elder \& K. Luscher (Orgs.), Linking lives and contexts: Perspectives on the ecology of human development (pp. 51-73). Cambridge: Cambridge University Press.

Mead, G. (1937). Cooperation and competition among primitive people. New York, NY: McGraw-Hill.

Mitjáns Martinéz, A. (1997). Criatividade, personalidade e educação. Campinas, SP: Papirus.

Morin, E. (1992). From the concept of system to the paradigm of complexity. Journal of Social and Evolutionary Systems, 15, 371-385.

Morin, E. \& Prigogine, I. (2000). A sociedade em busca de valores. Lisboa: Instituto Piaget.

Radke-Yarrow, M. \& Zahn-Waxler, C. (1991). The role of familial factors in the development of prosocial behavior: Research findings and questions. Em D. Olweus, J. Block \& M Radke-Yarrow (Orgs.), Development of antisocial and prosocial behavior (pp. 207-233). New York, NY: Academic Press.

Rogoff, B. (1990). Apprenticeship in thinking: Cognitive development in social context Oxford: Oxford University Press.

Siegler, R. S. \& Crowley, K. (1991).The microgenetic method: A direct means for studying cognitive development. American Psychologist, 46, 606-620.

Silva, N. (1999). Subjetividade. Em M. G. C. Jacques, M. N. Strey, M. G. Bernardes, P. A. Guareschi, S. A. Carlos \& T. M. G. Fonseca (Orgs.), Psicologia social contemporânea: Livro-texto ( $3^{\mathrm{a}}$ ed., pp. 168-180). Petrópolis, RJ: Vozes.

Shweder, R.(1990). Cultural psychology: What is this? Em J. W. Stigler, R. A. Shweder \& G. Herdt (Orgs.), Cultural Psychology (pp. 1-43).Cambridge, UK: Cambridge University Press.
Staub, E. (1989). Individual and societal (group) values in a motivational perspective and their role in benevolence and harmdoing. Em N. Eisenberg, J. Reykowski \& E. Staub (Orgs.), Social and moral values (pp. 45-57). Hillsdale, $\mathrm{NJ}$ : Lawrence Erlbaum.

Staub, E. (1991). A conception of the determinants and development of altruism and aggression: Motives, the self, and the environment. Em C. ZahanWaxler, E. M. Cummings \& R. Iannotti (Orgs.), Altruism and aggression: Biological and social origins (pp. 135-164). Cambridge, UK: Cambridge University Press.

Tappan, M. B. (1992). Texts and contexts: Language, culture, and the development of moral functioning. Em L. T. Winegar \& J. Valsiner (Orgs.), Children's development within social context (Vol. 1, pp. 93-117). Hillsdale, NJ: Lawrence Erlbaum.

Triands, H. C. (1991). Cross-cultural differences in assertiveness/competition vs group loyalty/cooperation. Em R. Hinde \& J. Groebel (Orgs.), Cooperation and prosocial behavior (pp. 78-88). Cambridge, MA: Cambridge University Press.

Triandis, H. C. (1995). Individualism and collectivism. San Francisco, CA: Westview Press.

Valsiner, J. (1987). Culture and the development of children's action: A theory of buman development. New York, NY: John Wiley \& Sons.

Valsiner, J. (1989). Human development and culture: The social nature of personality and its study. Lexington, MA: Lexington.

Valsiner, J. (1994). Bidirectional cultural transmission and constructive sociogenesis. Em W. de Graaf \& R. Maier (Orgs.), Sociogenesis reexamined (pp. 47-70). New York, NY: Springer.

Valsiner, J. (1998). The guided mind: A sociogenetic approach to personality. Cambridge, MA: Harvard University Press.

Valsiner, J. \& Cairns, R. (1992). Theoretical perspectives on conflict and development. Em C. U. Shantz \& W. W. Hartup (Orgs.), Conflict in child and adolescent development (pp. 15-35). Cambridge, MA: Cambridge University Press.

Valsiner, J., Branco, A. U. \& Dantas, C. (1997). Co-construction of human development: Heterogeneity within parental belief orientations. Em J. E. Grusec \& L. Kuczynski (Orgs.), Parenting and children's internalization of values (pp. 283-304). New York, NY: Wiley.

Van der Veer, R. \& Valsiner, J. (1988). Lev Vygotsky and Pierre Janet: On the origin of the concept of sociogenesis. Developmental Review, 8, 52-65.

Velho, G. (1987). Individualismo e cultura. Rio de Janeiro: Zahar.

Vygotsky, L. S. (1986). Thought and language (2 $\left.{ }^{\mathrm{a}} \mathrm{ed}\right)$. Cambridge MA: MIT Press.

Vygotsky, L. S. (1930 - 1994). The problem of environment. Em R. Van der Veer \& J. Valsiner (Orgs.), The Vygotsky reader (pp. 338-354). Oxford, UK: Basil Blackwell.

Wertsch, J. (1998). Mind as action. New York, NY: Oxford University Press.

\section{Sobre as autoras}

Recebido: 21/06/2002

Última Revisão: 08/09/2003

Aceite Final: 12/09/2003

Marilícia Witzler Antunes Palmieri é Professora Adjunto do Departamento de Psicologia Social e Institucional da Universidade Estadual de Londrina. É Doutora pela Universidade de Brasília.

Angela Uchoa Branco é Professora da Universidade de Brasília e responsável pelo Laboratório de Microgêneses das Interações Sociais. 